Thorax (1952), 7, 89.

\title{
OBSERVATIONS ON THE ANATOMY OF THE INTRASEGMENTAL BRONCHIAL TREE
}

\author{
BY \\ JOHN HAYWARD AND LYNNE McA. REID* \\ From the Royal Melbourne Hospital, Australia \\ (RECEIVED FOR PUBLICATION SEPTEMBER 24, 1951)
}

Miller's (1947) detailed description of the pulmonary lobule remains a classic, and the work of Nelson (1932, 1934), Foster-Carter (1942), and Brock (1946) on the larger bronchi and their distribution has firmly established the bronchopulmonary segment as a concept which forms a basis for localization of lung disease. But the bronchial tree between the segmental bronchi and the respiratory bronchioles has received less attention than its due. No clear description of its arrangement has been made.

An investigation, begun with the intention of studying the morbid anatomy of bronchiectasis, soon revealed the urgent need for further examination of this neglected region of the bronchial tree, because it is here that the dilatation of bronchiectasis occurs. The investigations described below were therefore carried out, and an attempt will be made in this paper to describe the intrasegmental bronchial tree and, on this basis, to give a method of localizing disease processes in this zone.

\section{Methods of Examination}

Observations were made on normal and pathological specimens and on bronchograms. In addition to 20 normal examples of each, operation specimens and bronchograms from 40 patients with cylindrical bronchiectasis were used. This was possible because in a study of bronchiectasis (Reid, 1950) it was shown that in this type of the disease the normal pattern of bronchial arborization is preserved. In these cases the bronchioles in the diseased segments fail to fill with oil. This makes their bronchograms particularly useful for observing the mode of branching of the bronchi, which are not obscured, as is so often the case in normal bronchograms, by the shadows of oil in overlying bronchioles and alveoli.

Immediately after removal from the body both operation and post-mortem specimens were fixed by inflation with $8 \%$ formol-saline through the bronchial tree, and before being cut they were left in the solution for seven to 10 days. The bronchi were exposed by cutting along their length with scissors. The side branches thus exposed could then in their turn be followed. It was sometimes necessary to pare away lung tissue to expose the branches more satisfactorily.

* Research Fellow, National Health and Medical Research Council. 
The 20 normal specimens were used to count the number of generations in the bronchial tree. Beyond the limit of visibility serial sections across the tubes were used to count as far as, but not including, the respiratory bronchioles. An average of three or four more generations of bronchioles were usually found in this way. In counting we called the segmental bronchus the first generation, the bronchi arising from its first division the second generation, and so on to the periphery.

We chose the posterior basal segment for our most exhaustive study, and only examined sufficient examples of other segments to show that the general pattern of bronchial arborization is the same in them all.

\section{DEFinitions}

In order that there shall be no ambiguity we now define certain terms used in this paper. The bronchial tree is considered to consist of two types of tubes, bronchi and bronchioles. We have followed Miller's definition that bronchi are the tubes which are proximal to the point at which cartilage disappears and bronchioles are those distal to this point in whose wall there is no cartilage. This study includes the terminal bronchioles which are those immediately proximal to the respiratory bronchioles. These latter are distinguished by the presence of alveolar openings along their walls.

When we speak of the route through the respiratory passages taken by air passing to or from a particular portion of the lung tissue we refer to it as a bronchial pathway. The portion of each air tube between successive subdivisions we call a bronchial or bronchiolar generation. Bronchial pathways are therefore made up of a series of bronchial and bronchiolar generations.

Each bronchopulmonary segment is irregularly pyramidal in shape, with its apex pointing towards the hilum. The segmental bronchus enters at the apex. In all segments it has been shown that the bronchial pathways with the most numerous generations fan out towards the periphery in directions deviating only slightly from the longitudinal axis of the segment and supply the alveoli at its distal surface. These have been called axial pathways, and the bronchi of which they are composed axial bronchi (Reid, 1950). The shorter bronchial pathways which pass from axial bronchi to the more proximal parts of the segment are called lateral pathways, and their bronchi lateral bronchi.

\section{OBSERVATIONS}

Arrangement of Intrasegmental Air Passages.-In each bronchopulmonary segment there are several axial pathways. As these continue to the surface most peripheral from the hilum, they continue so far into the segment, still with a considerable diameter, that they possess an individuality not shared by the lateral pathways. Among the multitude of bronchial divisions they can be clearly distinguished in both specimens and bronchograms, and they appear as a framework for the lung tissue which fills the space between and around them. They may be traced through bronchioles right to the periphery, where they end by dividing into terminal bronchioles which supply the lobules at the distal surface of the segment.

The side or lateral branches from axial pathways run a shorter course and soon divide into finer branches which fan out widely to supply the more proximal lung tissue in the segment. 
This arrangement of the air passages is seen in Fig. 1, which shows a bronchogram of a normal posterior basal segment. The segmental bronchus enters the top left-hand corner of the photograph. Its first branch, passing horizontally backwards, is usually regarded as the sub-apical segment of the lower lobe. Four or five axial bronchi are present, and the longest passes to the portion of the segment which fills the posterior costophrenic angle. Passing outwards from the segmental bronchus it is seen that at some points of division both the branches continue as axial pathways, and at others one is clearly a side branch which continues as a lateral pathway while the other proceeds axially.

To some extent the length of bronchial pathways is related to the distance of the lung tissue they supply from the segmental apex. In axial pathways the relation is fairly close. For instance, in the posterior basal segment illustrated in Fig. 1 it 'can be seen that the length of the pathways to lung tissue in the posterior costophrenic angle is greater than that to the remainder of the lung tissue resting on the diaphragm supplied by other axial bronchi. In the lateral pathways the relation is not as close, and it becomes less so the nearer the lung tissue is to the apex of the segment. This is because the lung tissue round the large proximal bronchi is not supplied directly from the bronchi it surrounds. It receives its air through lateral bronchi which arise from the axial bronchi more distally and send some of their branches back towards the apex. The result is that the proximal lung lobules receive their air by a circuitous route which is much longer than their direct distance from the apex.

The actual length of a bronchial pathway is impossible to measure with accuracy. During life it increases and diminishes with inspiration and expiration. After death shrinkage occurs when the lung is removed from the body, and still more with fixation. The shrinkage is probably greater in the peripheral part of the lung than in the proximal part, where the bronchi are supported by cartilage. The results of such measurement would be further invalidated by the impossibility in practice of accurately measuring a structure which does not always lie in one plane and is inevitably distorted by the dissection necessary to expose it. The number of subdivisions along a bronchial pathway is, however, related to its length and can be accurately counted. We use counts of the bronchial generations for indicating relative lengths of bronchial pathways and for specifying distances from the hilum along them.

Number of Subdivisions of the Bronchial TreE.-There is a variation in the number of generations in different pathways. We first determined the maximum number of generations we could trace in a given segment. The maximum number always occurred in the longest axial pathway to the most distal lung tissue of the segment. This figure was suitable for the comparison of different segments of the same lung and the same segment in different lungs.

The maximum figure we obtained for the posterior basal segment was between 20 and 25 generations from the segmental bronchus to the most distal terminal bronchiole inclusive. It was usually to the lung tissue in the costophrenic angle. In other segments there were slight variations which seemed to be related to their length. For instance, the lingula, which is a long segment, gave similar maximum figures, but in the apical segment of the lower lobe, which is shorter, the average 
figure was nearer 20. The variation in counts in corresponding segments of different adult lungs did not amount to more than several generations, and seemed to us within limits which might be regarded as the ordinary range of minor anatomical variation. Fig. 2 is a diagrammatic representation of the divisions along an axial pathway in a posterior basal segment. It shows 22 generations.

Most of our lungs were from adults. We studied insufficient lungs from children of various ages to be able to give definite figures, but our investigations did indicate that there were slightly fewer generations in children's lungs. This is consistent with the results of Willson (1928) and Palmer (1936). Palmer found an increase of three generations between birth and adulthood in the upper lobe.

The maximum number of generations along other axial pathways in a segment were found to be fewer than along the longest pathway, but once a deviation was made into lateral bronchi the count fell appreciably. Having passed into a lateral bronchus one could continue from then on to count the maximum number of generations by keeping to the axis of the subsegment. When this was done along successive lateral pathways from the hilum the total count from the segmental bronchus varied between about 15 and the number seen in axial pathways. This variation is in part due to the varying number of axial generations counted up to the point where the lateral pathway branches from the axial pathway. If their origin from the axial pathway to taken as the starting point, different lateral pathways give maximum counts which are much more nearly equal.

We also investigated the minimum number of generations which occurred and found that the smallest counts were obtained by tracing proximal side branches of proximal lateral bronchi leading back to lung tissue at the apex of the segment. Our lowest counts were between eight and 10. Thus the smallest number of bronchial generations which can occur between a segmental bronchus and a respiratory bronchiole is about eight and the largest about 25 .

Mode of Arborization in Bronchial and Bronchiolar Parts of the Bronchial TREE.-Early in our study of the bronchial tree we realized that it naturally divided itself into proximal and distal parts distinguished by the different shape and mode of branching of the tubes in the two parts. It was found that this variation corresponded with the division into bronchial and bronchiolar parts which was made on the basis of the presence of cartilage. The following differences can be observed.

Distribution of Subdivisions Along Bronchial Pathways.-The branches of the bronchial tree arise about three times closer to each other at the periphery than near the hilum. If an axial pathway is followed the average interval between branches for the first eight or 10 generations which comprise the bronchial part is about one centimetre. Over this length the points of division are usually very irregularly spaced. Often a few centimetres of bronchus are found free of branches, several of which then arise close together. In the bronchiolar part the subdivisions occur at fairly regular intervals of only a few millimetres until the pathway ends with a small cluster of terminal bronchioles which are even closer together (Fig. 2).

The bronchial part with its widely and irregularly spaced divisions occupies two-thirds to three-quarters of the length of the pathway, and the bronchiolar part with its more closely and regularly spaced divisions one-third to one-quarter. Lateral pathways are shorter and contain fewer divisions, but the relative spacing of the branches in each part is the same as in axial pathways. 


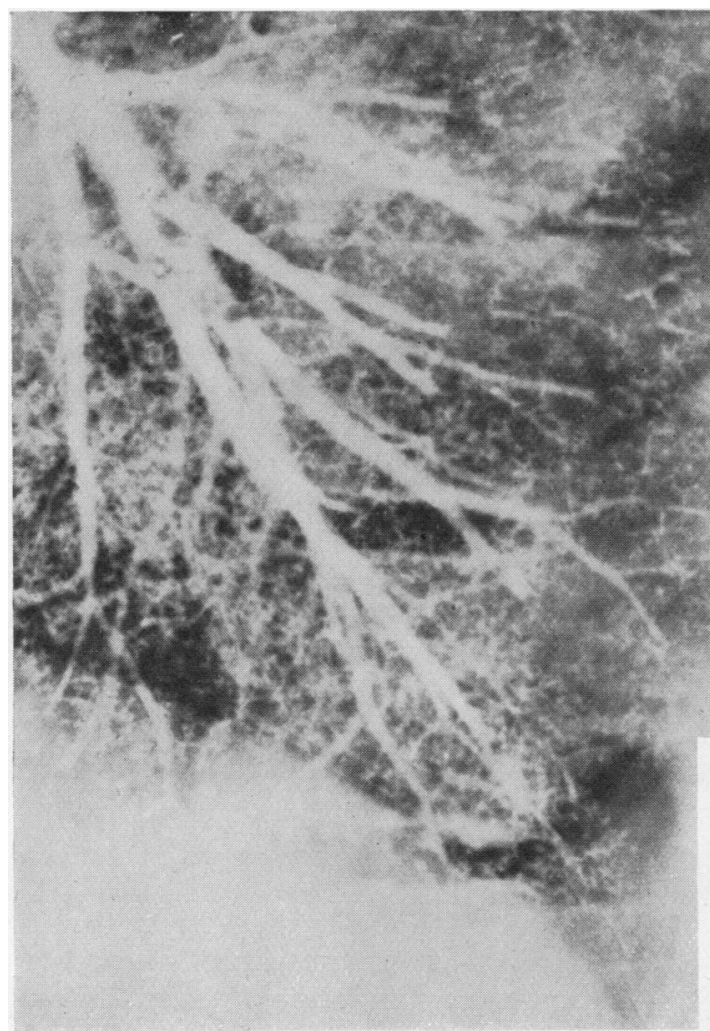

FIG. 1

FIG. 1.-Radiograph of the posterior basal bronchopulmonary segment. The segmental bronchus enters the photograph in the top left-hand corner. The "lateral branches" are seen passing mainly posteriorly while the axial pathways pass down towards the diaphragm.

FIG. 2.-Diagrammatic representation of the branches of an axial pathway. The segmental bronchus is considered the first generation, the bronchi arising from its first division the second generation, and so on down its length. The proximal 10 generations are drawn in a continuous black line to indicate there is cartilage present in the walls (bronchial part). The peripheral generations (bronchiolar part), from whose wall cartilage is absent, are drawn in a dotted line.

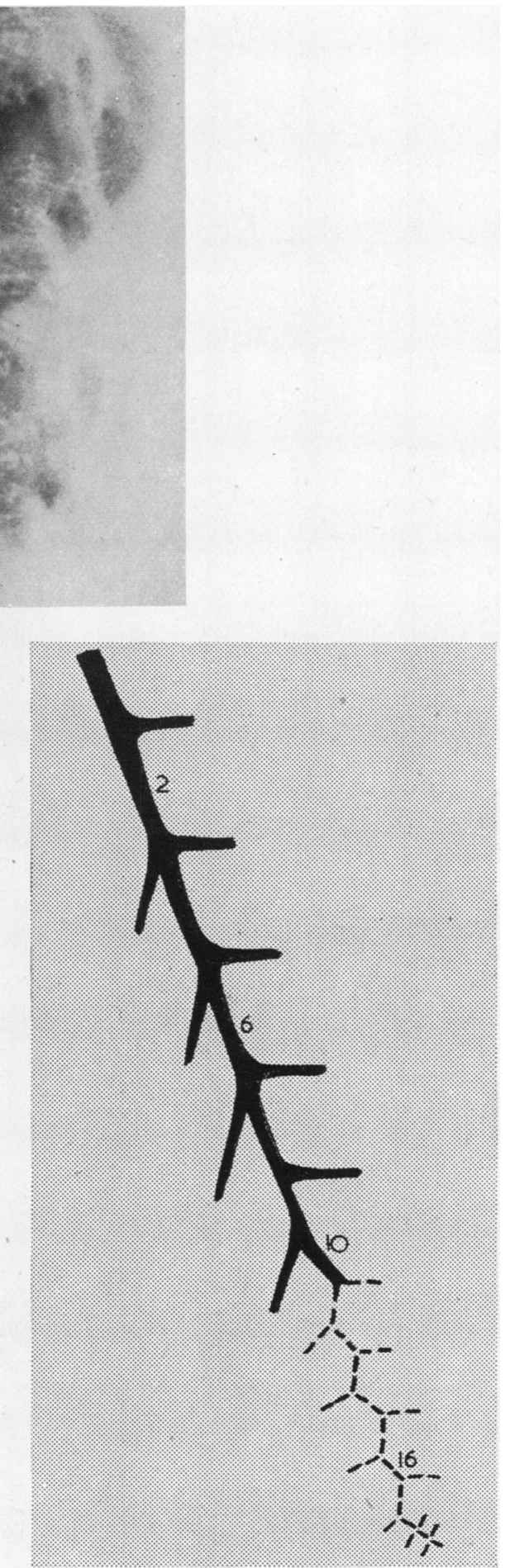

FIG. 2 
Modes of Branching.- In the bronchial part of the tree two sorts of division are seen. The more frequent is characterized by an acute angle and a well-defined carina between the branches. We have called divisions of this type "acute angle divisions," and we describe the branches arising from them as "acute angle branches." Often each branch arising from such a division appears to have an equal right to be regarded as the continuation of the main stem, and both may in fact lead to bronchial pathways of equal length and with a similar pattern of branching. In other cases one branch is larger and more in the direct line of the parent stem. This branch is then usually the continuation of an axial pathway while the other is the beginning of a shorter lateral pathway, but it may be only by tracing each branch to its destination that it can be certainly identified as an axial or lateral bronchus.

The less frequent type of bronchial division has an orifice which appears as a hole in the side wall of the parent stem by a wide angle, usually approximately a right angle, between the parent stem and the branch. There is no sharp carina. We have called these "wide angle divisions." At this type of division there is never any question which is the parent stem and which the branch so that, whereas at acute angle divisions the parent stem must often be regarded as breaking up into two acute angle branches, at wide angle divisions the parent stem retains its individuality and merely gives off one wide angle branch.

Axial pathways are made up exclusively of acute angle branches. Once a deviation is made into lateral pathways both acute and wide angle branches are found, the former leading particularly to the most distal part of the area of distribution and therefore being "axial" to the small subsegmental sector of lung tissue concerned, and the latter leading to more proximal parts and therefore being "lateral" in the sector.

Wide angle branches from axial pathways usually give rise within about a centimetre of their origin to a second wide angle branch which passes backwards towards the hilum to supply the lobules proximal to the lateral bronchus. We have called such branches " retrograde bronchi," because we believe that the fact that they" alone of all the bronchi drain in a direction opposite to the axial bronchi of the segment makes them worthy of distinction and may have some significance in reaeration of a massively collapsed lobe.

It may be mentioned here that the terms "wide" and "acute angle branches" can be applied to other than intrasegmental bronchi. The bronchus to the apical segment of the lower lobe and both upper lobe bronchi are wide angle branches, while the bronchi to the basal segments are acute angle branches.

In the bronchiolar part of the tree the mode of branching is different. The angle between divisions is always fairly wide, there is no true carina, and the branches appear as a hole in the side wall of the bronchiole. In this distal part of the tree the branches arise at approximately regular intervals. They are probably not all in the same plane as a diagram suggests. In radiographs a bronchiole often runs a zigzagging course. Each of the side branches of an axial bronchiolar pathway ends at once or after only a few further divisions in a similar cluster of terminal bronchioles.

Bronchioles are frequently visible in normal bronchograms, but it is scarcely ever possible to trace a bronchiolar pathway in its entirety. In films most suitable 
for clinical purposes with the bronchi clearly shown, bronchiolar filling is incomplete, and when the bronchioles are well filled overlying shadows of lipiodol in other tubes and in the alveoli obscure them.

Relative Diameters of the Tubes.-The bronchi taper from hilum to periphery, and in general their progressive narrowing is greatest near the hilum and becomes less rapid as they get smaller. The narrowing occurs mainly at points of division, rather than gradually all along the length. Portions of bronchi between divisions are almost exactly cylindrical. At every bronchial division each of the two branches is narrower than the parent stem, but the sum of their two diameters is greater than the diameter of the parent stem. Branches leading to lateral pathways are usually much narrower than the parent stem. This applies to all wide angle branches and to those acute angle branches which lead to lateral pathways. Acute angle branches which continue as axial bronchi are wider than adjacent lateral bronchi, and the bronchi of axial pathways are widest for the longest distances into the lung. It is therefore common for a peripheral generation of an axial pathway to be as wide as a proximal generation of a lateral pathway. The diameter of a bronchus at any point seems to be related to the volume of the lung tissue its branches supply and to the distance they have to cover to reach it.

The bronchioles are all of approximately the same diameter, which is of the order of a few millimetres. They taper only slightly towards the periphery.

\section{Discussion}

TERMINOLOGY.-The anatomists of the last century were impressed with the resemblance of the bronchial system to a tree, and took the analogy to the extreme of using botanical terms to describe the branching of the bronchus to each lung. In describing the intrapulmonary bronchi we have preferred to use the bronchopulmonary segments as the units because of their clinical and surgical significance. Within these we recognize axial and lateral pathways and have coined the two purely descriptive names "acute" and "wide angle" branches to describe the two structurally distinct types of bronchial subdivision which occur. The former have a carina, the latter have not.

Positions from Hilum to Periphery of Bronchial Tree.-When confronted with the problem of specifying distances out into the bronchial tree at which structures such as cartilage and mucous glands disappear from the walls of the air tubes, anatomists usually sidestepped it by recording the diameter of the tubes at which the change occurred. This is an unsatisfactory method of indicating the actual distance from the hilum, because it is using a variable measurement as a yardstick. Bronchial diameter fluctuates with each respiratory cycle during life, no doubt changes at death, and when measured in a specimen depends partly on the method of fixation and preparation. It is less in the child than in the adult, varies with the size of the individual, and is frequently altered by disease.

The statement that cartilage is absent in tubes of less than one millimetre may be useful when normal lungs are being described. However, such measurements of pathological conditions are not necessarily significant, nor do they enable comparison of healthy with diseased lungs. For example, the observation that in a bronchiectatic lobe cartilage disappears in bronchi of three millimetres in diameter would give no 
idea how far out in the bronchial tree the change occurred. It might even have occurred at the usual place, all the cartilage being anatomically intact and the higher figure being due to dilatation of the tubes at this level. By contrast, counts of bronchial generations are equally satisfactory both in anatomy and pathology. No matter how deformed the bronchi are by disease, so long as they are not completely destroyed their generations can be counted; and even when there is complete bronchial destruction the level beyond which this occurs can be determined by counting the bronchial generations between the hilum and the point of destruction. Even where there is deformity of the bronchial tree, as in saccular bronchiectasis, it is still possible to distinguish the axial pathways from the lateral as they are still in relation to the most distal surface of the segment.

There is a problem in specifying distances into the bronchial tree. Pathologists simply regard everything subpleural in position as peripheral in origin, an assumption not always justified, as will be shown. In recent years the importance of localizing lung lesions in terms of the bronchopulmonary segments involved has been accepted. The appreciation of the anatomy of pathological processes is an important early step in their study, and this localization of lung lesions in terms of bronchopulmonary segments led to great advances in pulmonary pathology and surgery. We believe that this other conception of localization in terms of distance from the hilum is equally important, especially for the fuller understanding of the pathology of conditions affecting the bronchial tree.

For instance, in different types of bronchiectasis the bronchi remain patent for a different number of generations from the hilum (Reid, 1950). Though the knowledge of which bronchopulmonary segments are affected by the disease is essential for planning its surgical removal, the localization of the level at which the bronchial lumen is obliterated or blocked by secretion is a necessary step in understanding its pathology.

The saccules in saccular bronchiectasis are subpleural. Consequently they have often been regarded as bronchiolar dilatations. This mistake would not have occurred had their distance from the hilum been measured in terms of the number of bronchial generations leading to the saccules. Reid (1950) showed that only three to five generations occur between the segmental bronchi and the saccules, thus proving that the saccules arise from lesions extending well into the proximal part of the bronchial tree and that their subpleural position is due to destruction of the bronchial tree and lung tissue distal to this level.

The following statements, which we believe to be correct, are further examples of the use of this method of localization. In massive collapse only about five proximal generations of segmental bronchi remain patent. In cylindrical bronchiectasis an average of about eight bronchial generations fills with lipiodol. An example from normal anatomy is that cartilage disappears from the walls of air passages at about the tenth generation in axial pathways.

In some cases it is useful merely to indicate which part of the bronchial tree is involved without bothering about actual counts. For example, in bronchiectasis the dilated tubes are nearly always confined to the bronchial part of the tree, and in bronchopneumonia the main lesions are in the bronchiolar part.

It is often illuminating to examine lesions in lungs, even when they appear to lie peripherally, with the conscious purpose of determining their connexion with the 
bronchial tree. This may require cutting along the bronchi rather than making the usual random slices with a large knife, disregarding the pulmonary architecture. In this way, tuberculomas are often found to lie immediately distal to a tuberculous stenosis of a fairly proximal bronchial generation. Lung cysts also repay this type of investigation. Thus, in a lobe with a localized area of mucous-filled cysts, it was shown that the cysts were dilated bronchi beyond an obstruction in the fourth generation of the segmental bronchial tree.

Enough examples have been given to illustrate this method of localization and its possibilities. Re-examination of familar things from a new viewpoint is frequently fruitful, and we believe that more careful study along these lines may lead to advances in pulmonary pathology.

\section{SUMMARY}

This paper describes observations on the anatomy of the intrasegmental bronchial tree which can be divided into a proximal bronchial part in which cartilage is present and a distal bronchiolar part from which cartilage is absent. In addition to this distinction the bronchi narrow progressively and have irregularly spaced branches of two types, acute angle divisions with a carina and less frequently wide angle divisions without a carina. The bronchioles taper only slightly and branch at regular intervals with all divisions similar. The branches have no carina and appear as a small circular hole in the side wall of the axial bronchiole.

Within any segment several axial pathways can be recognized which fan out from the apex to the most distal surface, thus forming a framework for the lung. Shorter lateral pathways arise from them and supply the more proximal parts of the segment. From the bronchial part which extends for about two-thirds of the length of an axial pathway branches arise. The remaining length is bronchiolar and from this 10 to 15 branches arise.

The importance of having a method of specifying positions in the bronchial tree from hilum to periphery is discussed, and it is suggested that this should be done by counting the bronchial generations from the hilum outwards. Examples of the application to pathology are given.

\section{REFERENCES}

Brock, R. C. (1946). The Anatomy of the Bronchial Tree. London.

Foster-Carter, A. F. (1942). Brit. J. Tuberc., 36, 19.

Miller, W. S. (1947). The Lung. Springfield, Ill.

Nelson, H. P. (1932). J. Anat., Lond., 66, 228.

- (1934). Brit. med. J., 2, 251.

Palmer, D. M. (1936). Amer. J. Anat., 58, 59.

Reid, L. McA. (1950). Thorax, 5, 233.

Willson, H. G. (1928). Amer. J. Anat., 41, 97. 